\title{
Impact of previous transurethral prostate surgery on health-related quality of life after radical prostatectomy: Does the interval between surgeries matter?
}

\author{
Michael Chaloupka ${ }^{1}$. Franka Figura ${ }^{1}$. Philipp Weinhold ${ }^{1}$. Friedrich Jokisch ${ }^{1} \cdot$ Thilo Westhofen $^{1} \cdot$ Paulo Pfitzinger $^{1}$. \\ Robert Bischoff ${ }^{1}$. Giuseppe Magistro ${ }^{1}$. Frank Strittmatter ${ }^{1} \cdot$ Armin Becker $^{1}$. Steffen Ormanns ${ }^{2} \cdot$ Boris Schlenker $^{1}$. \\ Alexander Buchner ${ }^{1}$. Christian G. Stief ${ }^{1} \cdot$ Alexander Kretschmer ${ }^{1}$ (i)
}

Received: 6 March 2020 / Accepted: 22 June 2020

(c) The Author(s) 2020, corrected publication 2021

\begin{abstract}
Purpose To assess the impact of previous transurethral surgery for benign prostate enlargement (BPE) and time interval between procedures on functional outcomes and health-related quality of life (HRQOL) after radical prostatectomy (RP). Methods A propensity score-matched patient cohort [ $n=685$, (513 without previous BPE surgery, 172 with BPE surgery)] was created and HRQOL was pre- and postoperatively assessed using validated questionnaires (EORTC QLQ-C30). Urinary continence was measured via ICIQ-SF questionnaire and pad usage. Multivariable analysis included binary logistic and Cox regression models $(p<0.05)$.

Results Median follow-up was 18 months. There was no significant difference in recurrence-free survival in multivariate analysis (HR 0.66, 95\% CI 0.40-1.07, $p=0.093$ ). We observe higher mean ICIQ-SF scores (5.7 vs. 8.2, $p<0.001)$ and daily pad usage $(1.3$ vs. $2.5, p<0.001)$, and decreased continence recovery (OR $0.46,95 \% \mathrm{CI} 0.30-0.71, p<0.001)$ for patients with BPE surgery. Postoperative general HRQOL scores were significantly lower for patients with previous BPE surgery (70.6 vs. $63.4, p=0.003$ ). In multivariate analysis, continence recovery (OR 5.19, 95\% CI 3.10-8.68, $p<0.001$ ) but not previous BPE surgery $(0.94,0.57-1.54, p=0.806)$ could be identified as independent predictors of good general HRQOL. There was no significant correlation between time interval between both surgeries and continence $(p=0.408)$, and HRQOL $(p=0.386)$ outcomes.

Conclusions We observe favourable continence outcomes for patients without previous BPE surgery. Our results indicate that RP can be safely performed after transurethral BPE surgery, regardless of the time interval between both interventions.
\end{abstract}

Keyword EORTC QLQ-C30 · Radical prostatectomy · Robot-assisted radical prostatectomy $\cdot$ Health-related quality of life $\cdot$ Transurethral resection of the prostate $\cdot$ Laser enucleation of the prostate $\cdot$ Laser vaporization of the prostate

\section{Abbreviations \\ BPE Benign prostate enlargement \\ GHS Global health status \\ HRQOL Health-related quality of life}

Michael Chaloupka and Franka Figura have contributed equally to this work.

Alexander Kretschmer

alexander.kretschmer@med.uni-muenchen.de

1 Department of Urology, Ludwig-Maximilians-University, Marchioninistrasse 15, 81377 Munich, Germany

2 Department of Pathology, Ludwig-Maximilians-University, Munich, Germany
ICIQ-SF International consultation on incontinence (short form)

ISUP International Society of Urological Pathologists

IIEF-5 International Index on Erectile Function

ORP Open radical prostatectomy

RARP Robot-assisted radical prostatectomy

RP Radical prostatectomy

TUR-P Transurethral resection of the prostate 


\section{Introduction}

Transurethral resection of the prostate (TUR-P) is the gold standard procedure in the management of refractory bladder outlet obstruction due to benign prostate enlargement, even though laser ablative transurethral procedures have become increasingly popular in recent years [1]. Radical prostatectomy (RP), on the other hand, represents an important cornerstone of the treatment of localized as well as locally advanced prostate cancer (PC) [2].

To date, there are data from several studies that investigated the effect of previous TUR-P on oncological as well as functional outcomes after RP, providing conflicting results [3-8]. In addition, generalizability of the current evidence is hampered by the fact that some studies analyzed historic cohorts $[3,4]$ or focused on a single surgical technique only $[3,4,7]$. Importantly, none of these studies evaluated healthrelated quality of life (HRQOL) and did not give information regarding the effect of the time interval between transurethral surgery and radical prostatectomy. To address these potential shortcomings, we created a large propensity scorematched cohort of contemporary patients that underwent RP in one experienced tertiary care centre and evaluated the impact of any type of previous transurethral BPE surgery as well as the time interval between both surgeries on patientreported outcomes with a focus on HRQOL.

\section{Patients and methods}

\section{Patient population, study design, and data assessment}

To be eligible for the current study, patients had to fulfil the following inclusion criteria: $\leq \mathrm{pT} 3$, no clinical lymphnode involvement, no clinical indication for metastatic disease based on preoperative bone scan or CT scan, and surgery performed by experienced surgeons with a minimum of 50 previous cases.

Between September 2013 and September 2019, 3436 radical prostatectomies $[n=2205$ open retropubic RP, $n=1231$ robot-assisted laparoscopic RP] have been performed in one tertiary care centre. After approval by an institutional review board, patient-reported outcomes were prospectively retrieved preoperatively as well as postoperatively. Hereby, questionnaires were sent per mail to eligible patients. Erectile dysfunction was assessed via the validated International Index of Erectile Function (IIEF5) questionnaire. Good erectile function was defined as IIEF-5 score of $\geq 18$.

Patients that met all inclusion criteria were retrospectively selected and, consequently, a propensity score matching including the variables "age at prostatectomy" as well as "prostate volume based on histopathological specimen", and "pT stage" was performed in a 1:3 fashion. Hereby, a matched cohort of 685 patients $(n=172$ with previous BPE surgery, $n=513$ without previous BPE surgery) was created and further analyzed.

Urinary continence was assessed using the International Consultation on Incontinence Questionnaire in its short form (ICIQ-SF). The ICIQ-SF is a three-item validated questionnaire. The total score ranges from 0 to 21 , with higher scores indicating greater severity of urinary incontinence [9].

HRQOL was assessed using the EORTC QLQ-C30 questionnaire. The primary endpoint "general HRQOL" was assessed based on the global health status (GHS) domain of the QLQ-C30 questionnaire (questions 29 and 30) following current EORTC instructions [10]. Following Snyder et al. [11], good general HRQOL was defined as GHS of $\geq 70$. For GHS, higher scores represent better general HRQOL. For QLQ-C30 functioning scores, higher scores represent a better functioning. For QLQ-C30 symptoms scores, higher scores represent greater impact of the respective symptom.

\section{Statistical analysis}

As indicated above, a propensity score matching was performed and a matched patient cohort was created. Comparisons of patient-reported outcomes as well as EORTC QLQ-C30 subdomains between both subgroups were performed using Kruskal-Wallis analysis of variance and post hoc testing whenever denoted. For categorical data, Fisher's exact test and Chi-square test were used. Primary endpoint for univariate and multivariable analyses was good general HRQOL at the respective time point based on a GHS score of $\geq 70$, following previously published cut-off values [11]. For multivariable analysis, binary logistic as well as Cox regression models were used. Here, the number of events was defined as the number of patients with a GHS score of 70 or more. For univariate survival analyses, Kaplan-Meier curves were generated and log-rank testing was performed. For correlation of continuous parameters, Spearman's rank correlation was used. All statistical analyses were performed using SPSS V26.0 (IBM, Armonk, NY, USA). A $p$ value of $<0.05$ was considered to be statistically significant.

\section{Results}

\section{Perioperative patient characteristics and oncological outcomes}

Detailed patient characteristics of the unmatched patient cohort are summarized in Table 1 . Briefly, mean prostate 
Table 1 Pre- and postoperative characteristics of patients included in the current study

\begin{tabular}{|c|c|c|c|c|c|c|}
\hline & \multicolumn{3}{|c|}{ Unmatched cohort } & \multicolumn{3}{|l|}{ Matched cohort } \\
\hline & No BPE surgery & BPE surgery & $p$ & No BPE surgery & BPE surgery & $p$ \\
\hline No. of patients & 3259 & 177 & & 513 & 172 & \\
\hline Age $(\text { years; mean } \pm S D)^{a}$ & $65.8 \pm 8.1$ & $66.5 \pm 7.9$ & 0.668 & $65.8 \pm 8.0$ & $66.4 \pm 8.0$ & 0.375 \\
\hline $\mathrm{BMI}\left(\mathrm{kg} / \mathrm{m}^{2} ;\right.$ mean $\left.\pm \mathrm{SD}\right)$ & $27.0 \pm 6.8$ & $26.7 \pm 3.1$ & 0.715 & $26.7 \pm 3.3$ & $26.4 \pm 3.3$ & 0.357 \\
\hline PSA preop. $(\mathrm{ng} / \mathrm{ml} ;$ mean $\pm \mathrm{SD})$ & $17.2 \pm 41.1$ & $15.8 \pm 23.2$ & 0.933 & $15.1 \pm 40.1$ & $12.9 \pm 17.5$ & 0.048 \\
\hline Prostate volume $(\mathrm{ml} ; \text { mean } \pm \mathrm{SD})^{\mathrm{a}}$ & $57.6 \pm 24.2$ & $49.4 \pm 14.2$ & 0.001 & $52.0 \pm 21.6$ & $50.2 \pm 20.6$ & 0.794 \\
\hline \multicolumn{7}{|l|}{ Gleason score $[n(\%)]$} \\
\hline 6 & $350(10.7)$ & $31(17.5)$ & 0.002 & $70(13.6)$ & $30(17.4)$ & 0.093 \\
\hline $7 \mathrm{a}$ & $1191(36.5)$ & $42(23.7)$ & & $191(37.2)$ & $46(26.7)$ & \\
\hline $7 b$ & $728(22.3)$ & $40(22.6)$ & & $109(21.2)$ & $39(22.7)$ & \\
\hline 8 & $394(12.1)$ & $19(10.7)$ & & $65(12.7)$ & $19(11.0)$ & \\
\hline 9 & $520(16.0)$ & $33(18.6)$ & & $72(14.0)$ & $32(18.6)$ & \\
\hline 10 & $47(1.4)$ & $6(3.4)$ & & $6(1.2)$ & $6(3.5)$ & \\
\hline \multicolumn{7}{|l|}{ pT stage $[n(\%)]^{\mathrm{a}}$} \\
\hline pT2 & $1868(57.3)$ & $98(55.4)$ & 0.512 & $280(54.6)$ & $94(54.7)$ & 1.000 \\
\hline pT3a & $710(21.8)$ & $34(19.2)$ & & $12624.6)$ & $41(23.8)$ & \\
\hline pT3b & $660(20.3)$ & $44(24.9)$ & & $107(20.9)$ & $37(21.5)$ & \\
\hline pT4 & $21(0.6)$ & $1(0.6)$ & & - & - & \\
\hline Lymph-node involvement [ $n(\%)]$ & $378(11.6)$ & $21(11.8)$ & 0.866 & $55(10.7)$ & $19(11.0)$ & 0.912 \\
\hline
\end{tabular}

$B P E$ benign prostate enlargement, $B M I$ body mass index, $P S A$ prostate-specific antigen, $S D$ standard deviation

${ }^{\text {a }}$ Propensity score-matched variables

volume was significantly higher for the patient subgroup without previous BPE surgery (57.6 vs. $49.4 \mathrm{ml}$, $p=0.001$ ).

In a next step, a matched cohort was created as described above. Detailed patient characteristics can be found in Table 1 . We did not find statistically significant differences regarding tumour stage $(p=1.000)$, age $(p=0.375)$, and prostate volume $(p=0.794)$. Of note, mean PSA levels were significantly higher in the subcohort of patients without previous $B P E$ surgery ( 15.1 vs. $12.9, p=0.048)$.

Median follow-up was 18 months (3-351) for the matched cohort. Follow-up was available for 127 (73.8\%) patients with previous BPE surgery and 379 (73.9\%) patients without previous BPE surgery. Regarding the diagnosis of PC, 120 (69.8\%) patients with previous BPE surgery were diagnosed by prostate biopsy, the remaining ones were scheduled for RP due to incidental PC diagnosis during the desobstruction procedure.

The operation time $(p=0.976)$ as well as intraoperative blood loss $(p=0.080)$ were not significantly different between both subgroups. In addition, positive surgical margin rate for pT2 and pT3 tumours did not differ significantly [31.6\% (no BPE surgery) vs. 34.5\% (BPE surgery)]. In multivariate Cox regression analysis stratified for Gleason score and positive surgical margin status, no statistically significant differences were observed for biochemical recurrencefree survival (HR 0.66 95\% CI 0.40-1.07, $p=0.093$; Fig. 1).



Fig. 1 Biochemical recurrence-free survival (bRFS) for patients with (red) and without (blue) previous transurethral surgery for benign prostate enlargement (BPE)

\section{Functional outcomes}

Preoperative as well as postoperative functional outcomes are summarized in Table 2. Briefly, we observed significantly higher preoperative rates of IIEF- 5 scores $\geq 18$ in $40.8 \%$ (no BPE surgery) vs. $29.1 \%$ (BPE surgery) of the patients $(p=0.018)$. Postoperatively, $12.9 \%$ vs. $6.3 \%$ of the respective patient subgroups had IIEF- 5 scores of $\geq 18$ ( $p=0.051)$. Regarding urinary continence, we found 
Table 2 Functional outcomes after a median follow-up of 12 months

\begin{tabular}{|c|c|c|c|c|c|c|}
\hline & \multicolumn{3}{|l|}{ T0 } & \multicolumn{3}{|l|}{ Follow-up } \\
\hline & No BPE surgery & BPE surgery & $p$ value & No BPE surgery & BPE surgery & $p$ value \\
\hline \multicolumn{7}{|l|}{ Erectile function } \\
\hline IIEF-5 score $($ mean \pm SD) & $11.2 \pm 10.1$ & $8.3 \pm 9.8$ & $<0.001$ & $5.7 \pm 7.7$ & $3.1 \pm 5.7$ & $<0.001$ \\
\hline IIEF-5 score $\geq 18(\%)$ & 40.8 & 29.1 & 0.018 & 12.9 & 6.3 & 0.051 \\
\hline \multicolumn{7}{|l|}{ Urinary continence } \\
\hline ICIQ-SF score $($ mean \pm SD $)$ & $0.9 \pm 2.5$ & $2.5 \pm 4.3$ & $<0.001$ & $5.7 \pm 5.2$ & $8.2 \pm 6.3$ & $<0.001$ \\
\hline Daily pad usage $($ mean \pm SD) & n.a & n.a & n.a & $1.3 \pm 1.9$ & $2.5 \pm 3.2$ & $<0.001$ \\
\hline Continence recovery $(\%)$ & n.a & n.a & n.a & 71.2 & 52.1 & 0.001 \\
\hline
\end{tabular}

$B P E$ benign prostate enlargement, ICIQ-SF International consultation of incontinence questionnaire short form, IIEF-5 international index of erectile function, $S D$ standard deviation

significantly higher mean ICIQ-SF scores (5.7 vs. 8.2, $p<0.001)$ and higher mean daily pad usage (1.3 vs. 2.5, $p<0.001)$ for patients with previous BPE surgery. Continence recovery, defined as use of up to one security pad per $24 \mathrm{~h}$, was reached by $71.2 \%$ in patients without previous BPE surgery, and $52.1 \%$ of patients with previous BPE surgery $(p<0.001)$. In multivariate analysis stratified for age and prostate volume, previous BPE surgery could be confirmed as an independent risk factor for decreased continence recovery (OR 0.46, 95\% CI 0.30-0.71, $p<0.001$ ).

\section{Health-related quality of life}

Health-related quality of life was assessed using the validated EORTC QLQ-C30 questionnaire. Analysis of functioning and symptoms subdomains as well as financial difficulties and general HRQOL based on GHS is summarized in Table 3. Preoperatively, no statistically significant differences in financial difficulty and functioning scales were observed. Regarding preoperative symptoms scale, there were significantly higher constipation scores in the BPE surgery subgroup (5.8 vs. $11.8, p<0.001)$. Based on GHS, $50.0 \%$ (previous BPE surgery) and $56.8 \%$ (no previous BPE

Table 3 Preoperative (T0) and postoperative health-related quality of life outcomes based on the validated QLQ-C30 questionnaire

\begin{tabular}{|c|c|c|c|c|c|c|}
\hline & \multicolumn{6}{|c|}{ Mean (SD) EORTC QLQ C30 score } \\
\hline & \multicolumn{3}{|l|}{ T0 } & \multicolumn{3}{|l|}{ Follow-up } \\
\hline & No BPE surgery & BPE surgery & $p$ & No BPE surgery & BPE surgery & $p$ \\
\hline \multicolumn{7}{|l|}{ Symptome scale } \\
\hline Dyspnoea & $9.3(20.2)$ & $6.6(19.2)$ & 0.061 & $14.1(25.8)$ & $10.5(21.1)$ & 0.318 \\
\hline Pain & $10.5(21.7)$ & $13.5(24.5)$ & 0.192 & $14.3(26.3)$ & $14.4(25.2)$ & 0.671 \\
\hline Fatigue & $16.8(24.5)$ & $16.0(21.0)$ & 0.846 & $24.0(25.7)$ & $26.0(23.3)$ & 0.216 \\
\hline Insomnia & $12.5(23.8)$ & $15.2(24.0)$ & 0.135 & $17.7(28.3)$ & $26.1(31.9)$ & 0.011 \\
\hline Appetite loss & $4.2(13.1)$ & $6.3(18.6)$ & 0.413 & $3.8(13.0)$ & $8.4(21.3)$ & 0.021 \\
\hline Nausea/vomiting & $1.1(5.5)$ & $1.1(5.7)$ & 0.803 & $2.3(8.0)$ & $1.8(5.7)$ & 0.893 \\
\hline Constipation & $5.8(17.8)$ & $11.8(22.9)$ & $<0.001$ & $12.0(23.4)$ & $13.7(25.5)$ & 0.593 \\
\hline Diarrhoea & $6.3(15.8)$ & $6.9(17.7)$ & 0.969 & $10.4(19.9)$ & $12.8(22.5)$ & 0.302 \\
\hline Financial difficulty scale & 4.5 (15.9) & $6.1(20.2)$ & 0.712 & $7.3(17.7)$ & $8.5(18.3)$ & 0.554 \\
\hline \multicolumn{7}{|l|}{ Functioning scale } \\
\hline Physical & $93.8(12.0)$ & $92.6(13.6)$ & 0.274 & $89.5(17.0)$ & $85.3(19.1)$ & 0.024 \\
\hline Role & $89.9(21.8)$ & $89.5(20.6)$ & 0.543 & $80.4(25.3)$ & $75.0(28.4)$ & 0.108 \\
\hline Cognitive & $88.8(17.9)$ & $86.8(20.8)$ & 0.629 & $82.6(22.3)$ & $83.9(20.1)$ & 0.716 \\
\hline Emotional & $73.8(23.4)$ & $74.5(23.9)$ & 0.696 & $77.1(24.4)$ & $70.5(25.5)$ & 0.013 \\
\hline Social & $84.8(23.3)$ & $84.5(21.8)$ & 0.616 & $75.9(26.7)$ & 69.4 (30.6) & 0.075 \\
\hline Global health status & $71.6(20.9)$ & $68.0(22.6)$ & 0.100 & $70.6(21.8)$ & $63.4(24.1)$ & 0.003 \\
\hline
\end{tabular}

$B P E$ benign prostate enlargement, $S D$ standard deviation 
surgery, $p=0.197$ ) could be classified as "good general HRQOL". We did not observe statistically significant differences in mean GHS scores between patients with and without previous BPE surgery (71.6 vs. $68.0, p=0.100$; Fig. 2, Table 3).

Postoperatively, analysis of the QLQ-C30 symptoms scale revealed significantly increased insomnia (17.7 vs. $26.1, p=0.011)$ and appetite loss scores (3.8 vs. 8.4, $p=0.021)$ for patients with previous BPE surgery compared to the subgroup without previous BPE surgery. In addition, emotional functioning scores (77.1 vs. 70.5, $p=0.013$ ) and physical functioning scores ( 89.5 vs. $85.3, p=0.024$ ) were significantly lower for patients with previous BPE

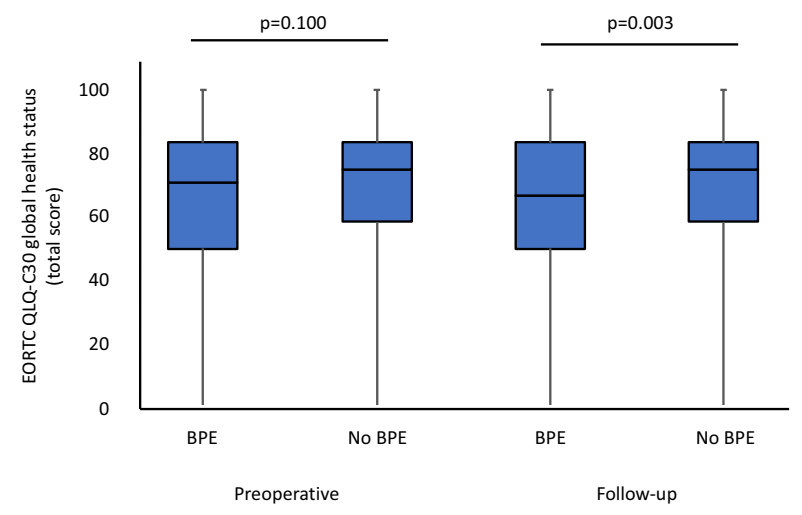

Fig. 2 General health-related quality of life based on the QLQ-C30 global health status pre- (T0) and postoperatively (BPE benign prostate enlargement) surgery. Postoperatively, mean GHS scores were significantly lower for patients with previous BPE surgery (70.6 vs. 63.4, $p=0.003$; Fig. 2, Table 3) with $40.8 \%$ (previous BPE surgery) and $52.0 \%$ (no previous BPE, $p=0.038$ ) being classified as "good general HRQOL" based on previously published GHS cut-off values [11]. Analysis of net changes of HRQOL subscales compared to baseline values is summarized in Table 4. Briefly, we observed no significant changes in GHS for the subgroup without previous BPE surgery $(-1.0, p=0.289)$. In contrast, a significant decrease for patients with previous BPE surgery $(-4.6, p=0.004)$ was found.

To address potential learning curve effects, a separate analysis was conducted for the two most experienced surgeons with $>1000$ previous RP vs. the remaining surgeons. Hereby, we did not find statistically significant differences regarding continence recovery $(74.4$ vs. $69.8 \%, p=0.410)$, IIEF-5 scores of $\geq 18$ ( 17.5 vs. $12.2 \%, p=0.321)$, and good general HRQOL based on QLQ-C30 GHS (46.8 vs. 49.4\%, $p=0.664$ ). In addition, we did not find significant differences in continence recovery $(p=1.000)$, erectile function recovery $(p=1.000)$, and good general HRQOL rates $(p=0.589)$ for patients who underwent previous HoLEP instead of TUR-P.

In multivariable analysis regarding the primary endpoint "good general HRQOL (defined as GHS score of 70 or more), we stratified BPE surgery subgroups by functional outcomes based on continence recovery and achievement of IIEF-5 scores of $\geq 18$ (Table 5). Hereby, continence recovery (OR 5.19, 95\% CI 3.10-8.68, $p<0.001$ ) but not previous
Table 4 Net differences between preoperative and postoperative health-related quality of life outcomes based on the validated QLQ-C30 questionnaire

\begin{tabular}{|c|c|c|c|c|}
\hline & \multicolumn{4}{|c|}{ Change in mean EORTC QLQ C30 scores from baseline } \\
\hline & No BPE surgery & $p$ & BPE surgery & $p$ \\
\hline \multicolumn{5}{|l|}{ Symptome scale } \\
\hline Dyspnoea & 4.8 & 0.001 & 3.9 & 0.008 \\
\hline Pain & 3.8 & 0.004 & 0.9 & 0.970 \\
\hline Fatigue & 7.2 & $<0.001$ & 10.0 & $<0.001$ \\
\hline Insomnia & 5.2 & 0.024 & 10.9 & $<0.001$ \\
\hline Appetite loss & -0.4 & 0.502 & 2.1 & 0.014 \\
\hline Nausea/vomiting & 1.2 & 0.028 & 0.7 & 0.463 \\
\hline Constipation & 6.2 & $<0.001$ & 1.9 & 0.088 \\
\hline Diarrhoea & 4.1 & 0.007 & 5.9 & 0.007 \\
\hline Financial difficulty scale & 2.8 & 0.030 & 2.4 & 0.257 \\
\hline \multicolumn{5}{|l|}{ Functioning scale } \\
\hline Physical & -4.3 & 0.001 & -7.3 & $<0.001$ \\
\hline Role & -9.5 & $<0.001$ & -14.5 & $<0.001$ \\
\hline Cognitive & -6.2 & $<0.001$ & -2.9 & 0.221 \\
\hline Emotional & 3.3 & 0.014 & -4.0 & 0.019 \\
\hline Social & -8.9 & $<0.001$ & -15.1 & $<0.001$ \\
\hline Global health status & -1.0 & 0.289 & -4.6 & 0.004 \\
\hline
\end{tabular}

$B P E$ benign prostate enlargement 
Table 5 Multivariable analysis regarding the primary endpoint, good general health-related quality of life (HRQOL)", defined as QLQ-C30 global health status score of at least 70

\begin{tabular}{lcccr}
\hline Predictive feature for good HRQOL & $\begin{array}{l}\text { Regression coef- } \\
\text { ficient }\end{array}$ & Odds ratio & $95 \%$ CI & $p$ value \\
\hline Previous BPE surgery (yes vs. no) & -0.62 & 0.94 & $0.57-1.54$ & 0.806 \\
IIEF-5 18 or more (yes vs. no) & 0.65 & 1.91 & $0.86-4.24$ & 0.110 \\
Continence recovery (yes vs. no) & 1.65 & 5.19 & $3.10-8.68$ & $<0.001$ \\
\hline
\end{tabular}

$B P E$ benign prostate enlargement, $C I$ confidence interval, $I I E F-5$ International index of erectile function questionnaire
BPE surgery $(0.94,0.57-1.54, p=0.806)$ could be identified as independent predictors of better postoperative general HRQOL.

\section{Time interval between transurethral BPE surgery and radical prostatectomy}

Information regarding the time interval between BPE surgery and RP was available for 144 of 172 patients (83.7\%). Median time interval between the BPE surgery and RP was 27 months (1-443). Time interval was $<12$ months for 56 patients (38.9\%) and $\geq 12$ months for 88 patients (61.1\%).

Using Spearman's rank correlation, we did not observe a significant correlation between time interval of transurethral BPE surgery and RP for urinary continence based on validated ICIQ-SF scores (correlation coefficient -0.082 , $p=0.408$; Fig. 3a) as well as for QLQ-C30 GHS scores (correlation coefficient $-0.084, p=0.386$; Fig. $3 b$ ). In line, we did not observe statistically significant differences in continence recovery rates between patients with less compared to more than 12 months of time interval between both surgeries (66.7 vs. $48.4 \%, p=0.131)$.

\section{Discussion}

In the current analysis, we provide data from a large contemporary propensity score-matched patient cohort and found favourable functional outcomes for patients that did not undergo previous BPE surgery and confirmed previous BPE surgery as an independent risk factor for decreased continence recovery in multivariable analysis. These findings are in line with results of recently published meta-analyses that found worse functional outcomes for patients with previous TUR-P [12, 13]. In the largest patient cohort to date, the authors found a significantly increased risk for urinary incontinence 3 months as well as 12 months after RP as well as worse erectile function recovery rates [8]. However, the continence rates that are reported in the current study have to be interpreted with caution. First, it has to be emphasized that the patient cohort analysed in the current study is a relatively high-risk patient population and a majority of patients were categorized in high-risk ISUP grade groups. This has
A



B

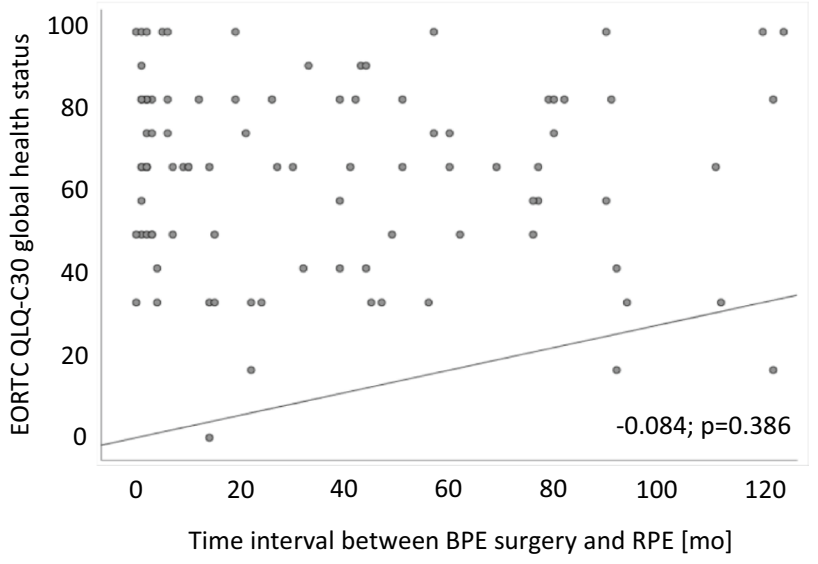

Fig. 3 Spearman's rank correlation regarding the impact of the time interval between transurethral benign prostate enlargement (BPE) surgery and radical prostatectomy (RPE) on urinary continence outcomes (a) and general health-related quality of life (b) (ICIQ-SF International consultation of incontinence questionnaire short form)

to be kept in mind when functional outcomes of the current study are compared with previously reported continence rates. In addition, definitions of continence as well as methods of continence function data retrieval vary between the currently available studies. In the current study, we provide continence data based on pad usage as well as the validated 
ICIQ-SF questionnaire to provide robust and reproducible continence data. Appropriate assessment of functional outcomes is important for preoperative patient counseling even if there are no adequate alternative therapies available, since it has been shown that well-perceived patient education has the potential to improve postoperative patient satisfaction following RP [14].

It has been postulated that increased inflammation and tissue fibrosis lead to more challenging surgical procedures, resulting not only in decreased functional but also oncological outcomes. Thus, it could be anticipated that a shorter period between BPE surgery and RP might lead to more difficult surgical procedures and ultimately leads towards decreased functional as well as oncological outcomes. For instance, Jaffe et al. analyzed the outcomes of 118 patients that underwent laparoscopic RP following TUR-P and found higher positive surgical margin rates for patients with previous TUR-P [3]. This is in line with the results of a recent meta-analysis by Li et al. [13]. However, there are also studies who failed to observe differences in positive surgical margin and biochemical recurrence rates [8]. Analogously, we did not observe any significant differences in positive surgical margin rates and biochemical recurrence-free survival for patients with previous BPE surgery in univariate and multivariate analysis.

In the absence of profound oncological data and conflicting results of functional outcomes, soft endpoints such as HRQOL are essential to assess potentially harmful effects of previous transurethral BPE surgeries. In addition to the results of previous studies, our study is the first to provide pre- as well as postoperative HRQOL data. For other urooncological entities, it has already been shown that preoperative HRQOL is an essential contributor in guidance of therapy decision-making [15] and it can be anticipated that this is also true for pre-RP patient counselling. While we observed significantly higher rates of good general HRQOL in univariate analysis, we were not able to confirm previous BPE as an independent risk factor for decreased general HRQOL in multivariate analysis stratified for continence recovery and IIEF-5 scores. Future studies with larger cohorts are, therefore, needed to confirm these preliminary results. In addition to general HRQOL scores, we provide detailed data from HRQOL subscales such as functioning and symptoms scores.

Another important novelty of the current study is the implementation of the time axis in the analysis of RP outcomes following transurethral BPE surgery. Interestingly, we did not observe any significant correlation between the time interval between BPE surgery and RP on continence as well as general HRQOL outcomes, and ICIQ-SF scores. While it can be hypothesized that inflammation is reduced over a longer time course, fibrosis-related effects might even increase within a longer time interval between both surgeries. Our results indicate that RP can be safely performed after transurethral BPE surgery, regardless of the time interval between both interventions.

The current study is not devoid of limitations. In addition to the limitations that are inherent to retrospective analyses in general, we use the non-prostate-specific EORTC QLQC30 questionnaire to address patients HRQOL. However, despite being not prostate cancer specific, this questionnaire provides robust results that can be compared with other entities as well as surgical procedures. To compensate the lack of domains that specifically address urinary and sexual symptoms, the validated IIEF-5 and ICIQ-SF questionnaires have been implemented in our analysis. Assessing HRQOL through validated questionnaires is advantageous in terms of generalizability and reproducibility of results but implies the important question whether statistically significant differences translate into clinically relevant differences. Even though this question cannot be adequately answered to date, a potential benefit of the QLQ-C30 questionnaire is that previously published and frequently used clinically relevant cut-off values are available to rely on [11]. Furthermore, the relatively short median follow-up of 18 months has to be addressed as another potential limitation of the current study and further studies with longer follow-up are needed in order to adequately address the impact of previous surgical desobstruction on biochemical recurrence-free survival after RP. In addition, it has to be emphasized that the subcohort with previous BPE surgery is still relatively small compared to their non-BPE surgery counterpart, which ultimately may lead to underpower and an increased probability of type II errors.

Finally, even though the current study is the first to report data from non-TUR-P surgically desobstructed patients, these subgroups are still small and larger, adequately powered studies are needed to further evaluate outcomes in this respective patient cohort.

\section{Conclusions}

In summary, we provide data from a large and well-balanced contemporary propensity score-matched patient cohort and focus on the impact of previous BPE surgeries on HRQOL outcomes following RP with an emphasis on the effect of the time interval between both procedures. After a median follow-up of 18 months, we found significantly decreased continence rates after previous BPE surgery as well as significantly higher postoperative general HRQOL scores for patients without BPE surgery in univariate analysis without statistically significant differences in multivariate analysis. We did not observe any significant impact of the time interval between both procedures indicating that RP can be safely performed regardless of the respective time interval. 
Author contributions MC, FF, RB, and AK: study design, data collection, data analysis, and manuscript writing/editing. PP, FJ, TW, FS, PW, GM, BS, and AB: data collection and manuscript editing. SO: data analysis and manuscript editing. AB: data collection/analysis and manuscript editing. CGS: protocol/project development, data analysis, coordination of study, and manuscript editing.

Funding Open Access funding enabled and organized by Projekt DEAL.

\section{Compliance with ethical standards}

Conflict of interest The authors declare that they have no conflict of interest.

Ethical approval This study was performed according to the $1964 \mathrm{Hel}-$ sinki Declaration and was approved by an institutional review board. Informed consent was obtained from all individuals participating in the study.

Open Access This article is licensed under a Creative Commons Attribution 4.0 International License, which permits use, sharing, adaptation, distribution and reproduction in any medium or format, as long as you give appropriate credit to the original author(s) and the source, provide a link to the Creative Commons licence, and indicate if changes were made. The images or other third party material in this article are included in the article's Creative Commons licence, unless indicated otherwise in a credit line to the material. If material is not included in the article's Creative Commons licence and your intended use is not permitted by statutory regulation or exceeds the permitted use, you will need to obtain permission directly from the copyright holder. To view a copy of this licence, visit http://creativecommons.org/licenses/by/4.0/.

\section{References}

1. Gravas S, Cornu JN, Gacci M, Gratzke C, Herrmann TRW, Mamoulakis C, et al. (2020) EAU Guidelines on Management of Non-Neurogenic Male Lower Urinary Tract Symptoms (LUTS), incl. Benign Prostatic Obstruction (BPO) 2020. European Association of Urology Guidelines 2020 Edition. Presented at the EAU Annual Congress Amsterdam 2020. European Association of Urology Guidelines Office, Arnhem

2. Mottet N, van den Bergh RCN, Briers E, Cornford P, De Santis M, Fanti S, et al. (2020) EAU-ESTRO-ESUR-SIOG Guidelines on Prostate Cancer 2020. European Association of Urology Guidelines 2020 Edition. Presented at the EAU Annual Congress Amsterdam 2020. European Association of Urology Guidelines Office, Arnhem

3. Jaffe J, Stakhovsky O, Cathelineau X, Barret E, Vallancien G, Rozet F (2007) Surgical outcomes for men undergoing laparoscopic radical prostatectomy after transurethral resection of the prostate. J Urol 178(2):483-487 (discussion 7)

4. Colombo R, Naspro R, Salonia A, Montorsi F, Raber M, Suardi N et al (2006) Radical prostatectomy after previous prostate surgery: clinical and functional outcomes. J Urol 176(6 Pt 1):2459-2463 (discussion 63)

5. Martin AD, Desai PJ, Nunez RN, Martin GL, Andrews PE, Ferrigni RG et al (2009) Does a history of previous surgery or radiation to the prostate affect outcomes of robot-assisted radical prostatectomy? BJU Int 103(12):1696-1698

6. Palisaar JR, Wenske S, Sommerer F, Hinkel A, Noldus J (2009) Open radical retropubic prostatectomy gives favourable surgical and functional outcomes after transurethral resection of the prostate. BJU Int 104(5):611-615

7. Hung CF, Yang CK, Ou YC (2014) Robotic assisted laparoscopic radical prostatectomy following transurethral resection of the prostate: perioperative, oncologic and functional outcomes. Prostate Int 2(2):82-89

8. Pompe RS, Leyh-Bannurah SR, Preisser F, Salomon G, Graefen M, Huland $\mathrm{H}$ et al (2018) Radical prostatectomy after previous TUR-P: oncological, surgical, and functional outcomes. Urol Oncol 36(12):527 e21-e28

9. Avery K, Donovan J, Peters TJ, Shaw C, Gotoh M, Abrams P (2004) ICIQ: a brief and robust measure for evaluating the symptoms and impact of urinary incontinence. Neurourol Urodyn 23(4):322-330

10. Aaronson NK, Ahmedzai S, Bergman B, Bullinger M, Cull A, Duez NJ et al (1993) The European Organization for Research and Treatment of Cancer QLQ-C30: a quality-of-life instrument for use in international clinical trials in oncology. J Natl Cancer Inst 85(5):365-376

11. Snyder CF, Blackford AL, Okuyama T, Akechi T, Yamashita H, Toyama $\mathrm{T}$ et al (2013) Using the EORTC-QLQ-C30 in clinical practice for patient management: identifying scores requiring a clinician's attention. Qual Life Res 22(10):2685-2691

12. Liao H, Duan X, Du Y, Mou X, Hu T, Cai T et al (2019) Radical prostatectomy after previous transurethral resection of the prostate: oncological, surgical and functional outcomes-a meta-analysis. World J Urol. https://doi.org/10.1007/s00345-019-02986-2

13. Li H, Zhao C, Liu P, Hu J, Yi Z, Chen J et al (2019) Radical prostatectomy after previous transurethral resection of the prostate: a systematic review and meta-analysis. Transl Androl Urol 8(6):712-727

14. Kretschmer A, Buchner A, Grabbert M, Sommer A, Herlemann A, Stief CG et al (2017) Perioperative patient education improves long-term satisfaction rates of low-risk prostate cancer patients after radical prostatectomy. World J Urol 35(8):1205-1212

15. Singh V, Yadav R, Sinha RJ, Gupta DK (2014) Prospective comparison of quality-of-life outcomes between ileal conduit urinary diversion and orthotopic neobladder reconstruction after radical cystectomy: a statistical model. BJU Int 113(5):726-732

Publisher's Note Springer Nature remains neutral with regard to jurisdictional claims in published maps and institutional affiliations. 